\title{
GESTIÓN DEL RIESGO DE DESASTRES, ORDENAMIENTO TERRITORIAL, REASENTAMIENTO Y REUBICACIÓN: CORRELACIÓN DESEQUILIBRADA PARA EL EJERCICIO DE DERECHOS HUMANOS DESDE RETROSPECTIVAS JURÍDICAS EN COLOMBIA
}

\author{
Jorge Eduardo Vásquez Santamaría \\ Martha Isabel Gómez Vélez \\ Hernán Darío Martínez Hincapié \\ Universidad Autónoma Latinoamericana \\ jorge.vasquez@unaula.edu.co - martha.gomez@unaula.edu.co - hernan.martinezhi@unaula.edu.co
}

\section{RESUMEN}

Como un avance sobre el problema de investigación ¿Cómo incide la gestión del riesgo de desastres por eventos naturales en Colombia en el ejercicio de los Derechos Humanos de las personas afectadas por procesos de reasentamiento poblacional? el cual apoya la sustentación de un Primer Informe de ampliación para la Comisión Interamericana de Derechos Humanos $(\mathrm{CIDH})$ después de celebrarse Audiencia Temática sobre las garantías de las poblaciones propensas a reasentamiento, esta ponencia presenta resultados de la relación entre el ordenamiento territorial y la gestión del riesgo a partir de retrospectivas construidas desde fuentes jurídicas y sociales. Hace una contextualización general del Primer Informe, devela aspectos relevantes de la evolución institucional y normativa de la gestión del riesgo de desastres como plataforma para el ejercicio de los Derechos Humanos, contrasta dicha evolución con el acumulado histórico derivado de los eventos catastróficos, ofreciendo un aporte crítico al orden jurídico institucional sobre las condiciones que la gestión del riesgo en el ordenamiento territorial ofrece para el ejercicio de los Derechos Humanos.

Palabras clave: gestión del riesgo de desastres, ordenamiento territorial, retrospectiva, Derechos Humanos.

\section{ABSTRACT}

As an advance on the research problem How does the risk management of natural disasters in Colombia affect the exercise of Human Rights of people affected by population resettlement processes? Which supports the presentation of a First Enlargement Report for the Inter-American Commission on Human Rights (IACHR) after the conclusion of the Thematic Hearing on the guarantees of populations prone to resettlement, this paper presents results of the relationship between land use and management of risk from retrospectives built from legal and social sources. It makes a general contextualization of the First Report, reveals relevant aspects of the institutional and normative evolution of disaster risk management as a platform for the exercise of Human Rights, contrasts this evolution with the historical accumulation derived from catastrophic events offering a critical input to the institutional juridical order on the conditions that the management of the risk in the territorial ordering offers for the exercise of Human Rights.

Key words: disaster risk management, land management, retrospective, Human Rights. 


\section{INTRODUCCIÓN}

Del Informe de Petición y la realización de una Audiencia Temática celebrada en Washington el 19 de marzo de 2015 ante la CIDH, sobre "Procesos de reasentamiento de población vulnerable en Colombia en el marco de procesos de reubicación de población desplazada, proyectos de desarrollo urbanístico, renovación urbana, y programas de prevención y atención del riesgo", se desprende el proyecto de investigación "Ejercicio de los Derechos Humanos en procesos de reasentamiento poblacional a partir de la gestión del riesgo de desastres por eventos naturales en Colombia". Esta investigación hace parte de un ejercicio de Enseñanza Clínica del Derecho desde la cual se despliega la estrategia de acción de la Clínica Jurídica de Interés Público de la Universidad Autónoma Latinoamericana (UNAULA) para sustentar los informes de ampliación sugeridos por la CIDH que buscan acrecentar el constructo argumentativo que soporta las aseveraciones contra el Estado de Colombia.

La investigación plantea como problema ¿Cómo incide la gestión del riesgo de desastres por eventos naturales en Colombia en el ejercicio de los Derechos Humanos de las personas afectadas por procesos de reasentamiento poblacional? el cual fue organizado en dos fases; en la primera, ya finalizada, se fijaron como objetivos específicos describir la evolución institucional y normativa, nacional e internacional, sobre la gestión del riesgo de desastres como plataforma jurídica y política del ejercicio de los Derechos Humanos de poblaciones propensas a procesos de reasentamiento; sistematizar las principales afectaciones a los Derechos Humanos padecidas por poblaciones en el territorio nacional entre el 2000 y 2014 a partir de los datos oficiales de la institucionalidad constituida para la gestión del riesgo de desastres; valorar la incidencia de la gestión del riesgo de desastres por eventos naturales en Colombia sobre el ejercicio de los Derechos Humanos de las poblaciones afectadas por procesos de reasentamiento; y construir una recomendación a partir de la cual sea posible subsanar debilidades en la gestión del riesgo de desastres para la garantía y protección del ejercicio de Derechos Humanos.

Como resultado de la primera fase, el Primer Informe reúne una retrospectiva sobre la gestión del riesgo de desastres por evento natural, toda vez que fuera de ser una de las variables propuestas a la CIDH, es una materia legalmente imperativa para el ordenamiento territorial a cargo de departamentos y municipios en Colombia. Para ello se realizó una descripción valorativa jurídica e institucional, nacional e internacional, que se posicionó como plataforma dogmática para la evaluación del contexto situacional que ha determinado el ejercicio de los Derechos Humanos en poblaciones propensas a programas y procesos de reasentamiento en los últimos quince años. Luego profundiza en el análisis de las secuelas humanas en el territorial nacional, construyendo una retrospectiva que sistematiza en los últimos quince años los consolidados departamentales relacionados con los muertos, heridos, desparecidos, personas y familias registradas como víctimas provocadas por trece modalidades de evento natural, ofreciendo un consolidado estadístico de gestión de riesgos de desastres con base en la información recopilada por la Unidad Nacional de Gestión del Riesgo de Desastres (UNGRD), para finalizar con unas valoraciones y recomendaciones a las autoridades nacionales. Todos los componentes citados integran el Primer Informe de ampliación para la $\mathrm{CIDH}$, denominado "Retrospectivas sobre la gestión del riesgo en Colombia como panorama general para el ejercicio de los Derechos Humanos en los procesos de reasentamiento de población vulnerable".

Como resultado adscrito al Primer Informe, este trabajo expone la evolución de la relación entre el ordenamiento territorial y la gestión del riesgo a partir de retrospectivas construida desde fuentes jurídicas y sociales, inicialmente desde aspectos institucionales y normativos, y luego desde el acumulado histórico derivado de los eventos catastróficos ocurridos en los treinta y dos departamentos de Colombia en los últimos quince años, para finalizar con algunas reflexiones sobre las condiciones que ofrece la gestión del riesgo en el ordenamiento territorial para el ejercicio de los Derechos Humanos.

\section{DEL CONTEXTO PROBLemATIZADOR DE LA GESTIÓN DEL RIESGO DE DESASTRES POR EVENTOS NATURALES}

El abordaje de la gestión del riesgo de desastres naturales como una de las dimensiones que desencadena procesos de reasentamiento poblacional en Colombia parte de condiciones fácticas que contextualizan las complejas circunstancias de grupos poblacionales propensos a reubicación territorial. 
La estratégica posición geográfica de Colombia permite que el territorio nacional sea catalogado como de incidencia andina debido a la participación montañosa que define la zona del interior; de incidencia amazónica por la participación en la zona selvática del sur, y de incidencia pacífica y atlántica debido a la participación sobre los dos océanos. La concurrencia de distintos ecosistemas, pisos térmicos y climas en una fracción tan concreta del territorio Latinoamericano desata la presencia de distintos eventos naturales que se alteran e intensifican, sea por fenómenos aparentemente conocidos como los del calentamiento oceánico, o por otros no tan conocidos como el cambio climático, lo que hace de Colombia un país propenso y vulnerable a la ocurrencia de eventos naturales.

A lo anterior se suma una tradición de organización demográfica en el territorio que se aparta de la experiencia Latinoamericana. Como se aprecia en Viviescas (1989) Zambrano (2013) Morcillo Dosman (2007) y Sánchez Steiner (2008), esta organización se determinó por procesos fundacionales de ciudades, posteriormente influidos por migraciones causadas por los cambios en los modelos económicos acogidos por el Estado y los conflictos internos, resultando un país con fuertes episodios de desplazamiento que acentuaron el crecimiento de la urbanización en múltiples concentraciones urbanas, sin que se configurara un prototipo de único centro de impulsión económica, política y cultural para el territorio nacional, y por el contrario se afianzara más un modelo de regionalización desarrollado por la normativa de manera tímida.

Los eventos naturales que por su intensidad y frecuencia pueden ser vistos en ocasiones como bondades del territorio gracias a la movilización de procesos que proporcionan servicios ambientales, se constituyen también en una variable generadora de riesgo y catástrofes, lo que empeora si se tiene presente la diversidad territorial y ecosistémica en la que grupos sociales diversos culturalmente habitan las distintas regiones, en las cuales la construcción del territorio se manifiesta con el crecimiento de ciudades y pueblos, la expansión de proyectos agropecuarios que prolongan la frontera agrícola, explotaciones mineras y proyectos de infraestructura.

Además el proceso de urbanización del territorio ha generado en las últimas décadas gran cantidad de reasentamiento de población, donde las condiciones de habitabilidad no son subsanadas con los desplazamientos y su posterior reasentamiento, en la medida que muchas personas salen de zonas de riesgo por evento natural para asentarse en otras que si bien cuentan con características diferentes, son igualmente riesgosas y propensas a los desastres causados por eventos naturales; como lo expresan Victoria Morales y Molina Prieto (2003): "La ciudad colombiana es la expresión de un crecimiento continuo de población, que se va acrecentado periódicamente por fenómenos migratorios debidos a grandes desplazamientos, generados por desastres naturales, violencia, conflicto armado o por empobrecimiento de zonas rurales (búsqueda de mejores condiciones de vida)".

A pesar de su creciente frecuencia e intensidad, el riesgo de desastres por evento natural es una variable poco explorada e indagada, pero desde ella se movilizan interesantes procesos en el ámbito normativo, internacional, judicial y de políticas públicas que reclaman del Estado una nueva perspectiva del cumplimiento de su función pública en relación con la gestión del riesgo y los procesos de reasentamiento y reubicación. Como lo expresa el Banco Mundial (2011: 35) cuando explica el reasentamiento en el marco de una política integral de gestión del riesgo: "la falta de la planificación en el uso del suelo para orientar la localización de asentamientos humanos, la debilidad institucional para aplicar las normas al respecto, cuando éstas existen, y la falta de programas de vivienda para población de bajos recursos, lleva a que asentamientos humanos se localicen en zonas de riesgo."

El Primer Informe de ampliación a la CIDH adiciona a la problematización el negligente tratamiento de parte de la estructura administrativa para la gestión del riesgo de desastres, al que se suma el incremento de procesos de reasentamiento poblacional en los cuales se mantiene o agrava el ejercicio de los Derechos Humanos debido a la tendencia reactiva, mediática e improvisada de muchas autoridades competentes. Sin que sea la mejor opción para estas variables del problema, se exalta que la falta de un marco normativo nacional - legal y reglamentario - que norme de forma integral y completa el proceso de reasentamiento y reubicación poblacional en casos de desastre y catástrofe por eventos naturales, dilata y obstaculiza los procesos de reglamentación a cargo de las entidades territoriales, y desde ellos, la formulación y ejecución de políticas públicas con proyección y continuidad, entidades 
territoriales que desde el poder de autonomía que adquieren en la descentralizadas administrativa, generan procesos diversos y no uniformes que parecen desnaturalizar estándares de tratamiento igualitario en la población afectada por eventos naturales. Lo anterior también mantiene las necesidades y falencias en los sistemas de planeación, específicamente desde la funcionalidad y eficacia de varios de los instrumentos técnico - normativos como los Planes de Ordenamiento Territorial y los Planes de Desarrollo, de los cuales se evidencia negligencia frente a la actualización y pertinencia de los estudios que deben transversalizar aquellos instrumentos como medios de prevención y garantía para el ejercicio de los Derechos Humanos de la población en el territorio.

\section{RELACIÓN DEL ORDENAMIENTO TERRITORIAL Y LA GESTIÓN DEL RIESGO DESDE LAS RETROSPECTIVAS JURÍDICO INSTITUCIONALES}

Desde el objetivo específico encaminado a describir valorativamente la evolución institucional y normativa, nacional e internacional, de la gestión del riesgo de desastres como plataforma jurídica y política del ejercicio de los Derechos Humanos de poblaciones propensas a procesos de reasentamiento, el Primer Informe a la $\mathrm{CIDH}$ ofrece varias premisas que permiten caracterizar la relación entre el ordenamiento territorial y la gestión del riesgo de desastres, y desde ella, valorar su incidencia en el ejercicio de los Derechos Humanos.

Primero se hace posible asegurar que la gestión del riesgo de desastres, el ordenamiento territorial, y el reasentamiento poblacional, han sido materias que dentro de la evolución jurídico institucional colombiana no surgieron articuladas, por el contrario, ha sido su progresiva evolución, nacional e internacional, la que ha impulsado la correlación entre las mismas. Segundo, si bien esa correlación hoy cuenta con un respaldo normativo e institucional, la misma no es equilibrada y armónica, lo que devela una mayor y progresiva articulación nominal entre el ordenamiento territorial y la gestión del riesgo de desastres, y no con el reasentamiento poblacional, lo que se evidencia en el caso colombiano con la necesidad de regular esta última materia ante la ausencia de una normativa integral. Tercero, la desarticulación normativa e institucional en el surgimiento de las tres figuras y su correspondiente correlación desequilibrada, encuentra en la visión paradigmática que pregonó el goce a un ambiente sano, la razón a partir de la cual la gestión del riesgo tuviera aparición y desarrollo, la misma que luego impactó e incidió en el ordenamiento territorial pero que no ha hecho lo propio con el reasentamiento y la reubicación poblacional. A partir del ambiente sano se han promovido enfoques para el ejercicio de la función administrativa a cargo de las autoridades competentes a través de la estructuración y funcionalidad de SNGRD, regulaciones para el ordenamiento territorial y la planeación, como la promoción de una cultura jurídico política de participación social responsabilizada y empoderada para el ejercicio de Derechos Humanos de los cuales son titulares. Comenzaremos desarrollando la última premisa.

Evidenciar que las preocupaciones que impulsan las condiciones para garantizar el goce de un ambiente sano promovieron la aparición y evolución de las otras figuras para nada es una premisa negativa; distinto a las formas en cómo se empleó dicha garantía en el surgimiento y correlación que la gestión del riesgo de desastres, el ordenamiento territorial y el reasentamiento y reubicación tuvieron dentro del ordenamiento jurídico e institucional. Fijando como punto de llegada en la reconstrucción normativa e institucional al SNGRD, y en él, a la UNGRD como fuente principal de la información de víctimas generadas a raíz de eventos naturales, la reconstrucción de las normas adoptadas por el Estado en la materia para asegurar el ejercicio de los Derechos Humanos, y con ello, de la relación entre la gestión del riesgo de desastres con las otras figuras, inicia en los años setenta por las siguientes razones.

La gestión del riesgo se ha vinculado con la ocurrencia de desastres de tipo natural, ámbito especialmente normado en Colombia a partir de la ya referida década, cuando comienzan a fortalecerse y proliferar disposiciones normativas internacionales referentes al medio ambiente, lo que incide en la regulación y reglamentación de acontecimientos o fenómenos directamente vinculados con causas naturales, lo que asocia la ocurrencia de estos eventos a condiciones ambientales; ejemplo de ello son algunas disposiciones del Decreto 1355 de 1970, el Decreto 2811 de 1974 y de la Ley 9 de 1979, normativa fuertemente incidida y determinada por disposiciones internacionales. 
En la Declaración de las Naciones Unidas sobre el Medio Humano de Estocolmo 1972, se reconoció como principio de convicción común para los Estados el siguiente: "Como parte de su contribución al desarrollo económico y social, se debe utilizar la ciencia y la tecnología para descubrir, evitar y combatir los riesgos que amenazan al medio, para solucionar los problemas ambientales y para el bien común de la humanidad". De allí que la Declaración de 1972 se traduzca en un referente incidente en el desarrollo jurídico en materia ambiental, y de manera especial en la gestión del riesgo de desastres, pues si bien es un conjunto de disposiciones de naturaleza lata o flexible a la luz del artículo 93 de la Constitución Política de 1991, su trascendencia en el ordenamiento jurídico nacional es recalcada por la jurisprudencia de la Corte Constitucional, como sucede en las sentencias C - 595 de 2010, en la que se emplea como parámetro que explica el surgimiento del Derecho ambiental en décadas recientes, la T 411 de 1992 y T - 724 de 2011 como parte justificante de la aparición de la Constitución Ecológica de 1991 en Colombia, Norma suprema que en el artículo 80 contempla como una de las obligaciones especiales del Estado "prevenir y controlar los factores de deterioro ambiental".

La misma Declaración de 1972, al igual que el artículo 80 constitucional, fija la planificación ambiental como un deber para el Estado, desde el cual, se hacía posible elaborar una plataforma cultural para generar un desarrollo aproximado a la sostenibilidad a partir de una previsión sistemática en lo organizacional, funcional, administrativo e incluso en lo participativo. Ello se desprende de los principios trece, donde se dispuso "A fin de lograr una más racional ordenación de los recursos y mejorar así las condiciones ambientales, los Estados deberían adoptar un enfoque integrado y coordinado de la planificación de su desarrollo, de modo que quede asegurada la compatibilidad del desarrollo con la necesidad de proteger y mejorar el medio ambiente humano en beneficio de su población.", lo que no solo sigue impregnando las disposiciones jurídicas vigentes sobre los instrumentos de planeación en las diferentes escalas administrativas que tienen la obligación de incorporar la gestión del riesgo, sino que busca ilustrar las orientaciones que en materia política debe incluir la gestión del riesgo como un insumo para equilibrar las demandas del desarrollo con protección y mejoramiento del medio ambiente, lo que en últimas exige inversión en gestión del riesgo en términos de conocimiento preventivo de los ecosistemas y recursos en los territorios.

El principio catorce dispone que "La planificación racional constituye un instrumento indispensable para conciliar las diferencias que puedan surgir entre las exigencias del desarrollo y la necesidad de proteger y mejorar el medio ambiente." lo que debe significar acciones para la comprensión de las condiciones y variables territoriales en la Nación, permitiendo la adopción de estrategias diferenciales que respondan de manera particular, concreta e integrada a una planificación protectora de Derechos Humanos. El principio quince cobra especial importancia, en la medida que contempla aplicar la planificación "a los asentamientos humanos y a la urbanización con miras a evitar repercusiones perjudiciales sobre el medio ambiente y a obtener los máximos beneficios sociales, económicos y ambientales para todos.", fijando un soporte para combatir uno de los fenómenos, sino el más incidente, de la generación de vulnerabilidad al riesgo del desastre, como es el crecimiento de los centros urbanos caracterizados por procesos demográficos complejos que hacen del urbanismo una tarea prioritaria para las agendas de gobierno. A su finalidad se suma el sentido del principio dieciséis: "En las regiones en que exista el riesgo de que la tasa de crecimiento demográfico o las concentraciones excesivas de población perjudiquen al medio ambiente o desarrollo, o en que la baja densidad de población pueda impedir el mejoramiento del medio ambiente humano y obstaculizar el desarrollo, deberían aplicarse políticas demográficas que respetasen los derechos humanos fundamentales y contasen con la aprobación de los gobiernos interesados."

En la Carta Mundial de la Naturaleza de 1982, adoptada por la Asamblea General de las Naciones Unidas mediante Resolución 37/7, dispone entre las funciones a los Estados que "En la planificación y realización de las actividades de desarrollo social y económico, se tendrá debidamente en cuenta el hecho de que la conservación de la naturaleza es parte integrante de esas actividades", lo que para el caso del Estado de Colombia, incluso a partir de la legislación vigente para la época, debía representar mínimamente la incorporación de la gestión del riesgo de desastres en los planes de desarrollo como instrumentos de planeación. Seguidamente prevé que "Al formular planes a largo plazo para el desarrollo económico, el crecimiento de la población y el mejoramiento de los niveles de vida se tendrá debidamente en cuenta la capacidad a largo plazo de los sistemas naturales para asegurar el 
asentamiento y la supervivencia de las poblaciones consideradas, reconociendo que esa capacidad se puede aumentar gracias a la ciencia y la tecnología", derrotero de singular importancia en la gestión del riesgo, toda vez que reitera y actualiza los cometidos de Estocolmo de 1972, en el sentido de contar con un suficiente y acreditado reconocimiento y comprensión de las características y condiciones de los componentes naturales y ecosistémicos del territorio como lineamiento para la ejecución de políticas destinadas a garantizar el bienestar de la población, aspecto que de efectuarse a cabalidad, se traduciría en localización adecuada de asentamientos humanos, tanto urbanos como rurales, capacidad de recursos in situ para la prestación de servicios ambientales, reconocimiento y comprensión de factores de vulnerabilidad, y minimización de intervenciones con impacto para satisfacción de necesidades de los conglomerados poblacionales. Finalmente dispuso la "asignación de partes de la superficie terrestre a fines determinados y se tendrán debidamente en cuenta las características físicas, la productividad y la diversidad biológica y la belleza natural de las zonas correspondientes", dimensión que hoy justifica la declaratoria de suelos de protección cuando reúnan características de riesgo no mitigable.

Como se aprecia de las disposiciones internacionales citadas, hasta finales de la década de los ochenta la gestión del riesgo de desastres se desarrolló como un apéndice del sector ambiental, recolectando figuras de especial relevancia como la valoración de los recursos ambientales, la planificación, y los procesos demográficos y urbanos en el territorio. Si bien este compendio de disposiciones tienen en la actualidad un profundo eco en el nuevo orden constitucional de Colombia, en aquella época igualmente promovieron la aparición de normas como las propias de los ya citados Decreto 1355 de 1970, el Decreto 2811 de 1974 y la Ley 9 de 1979. No obstante, vale reiterar que si bien el tratamiento que recibió la gestión del riesgo de desastres por parte del ordenamiento jurídico nacional soldó una correlación de esa materia con el goce a un ambiente sano, la misma se dio en términos donde con esfuerzo se lograba particularizar la naturaleza y alcance propio de la gestión del riesgo, al estar subsumida en las apuestas ambientales internacionales que impactaron el orden interno. Lo anterior mantuvo una visión globalizante de ambas materias pero con una relación jerárquica donde la gestión del riesgo se leyó primordialmente en términos de protección a la vida, la salud, la salubridad pública y el saneamiento básico, sin que sobresaliera en ella una identidad preventiva y resiliente enfocada al ordenamiento del territorio.

Para la década de los años noventa la gestión del riesgo de desastres define un sendero que sin contrariar los avances en materia ambiental, precisa un desarrollo jurídico y político independiente impulsado principalmente por la ONU. Con la Resolución 44/236 de 1989 se acogió el Marco Internacional de Acción para el Decenio Internacional para la Reducción de los Desastres Naturales, a partir del cual la Asamblea General de la ONU vio la necesidad de mejorar la eficiencia de los esfuerzos colectivos internacionales en la prestación de asistencia humanitaria debido a la preocupación de la situación de las víctimas de desastres y situaciones de emergencia. Más tarde la Resolución 46/182 de 1991 establece el "Fortalecimiento de la coordinación de la asistencia humanitaria de emergencia de las Naciones Unidas", y con la Declaración de Rio de Janeiro de 1992, se proporciona un marco motivo integral desde los 27 principios que la conforman, pero de manera especial, a diferencia de las disposiciones de las décadas anteriores, esta Declaración define el deber de "(...) notificar inmediatamente a otros Estados de los desastres naturales $u$ otras situaciones de emergencia que puedan producir efectos nocivos súbitos en el medio ambiente de esos Estados. La comunidad internacional deberá hacer todo lo posible por ayudar a los Estados que resulten afectados.". La Declaración no fue omitida por el Estado colombiano, y por el contrario, fuera de impactar el ordenamiento jurídico e institucional interno, seria promotora de un desarrollo especializado de la gestión del riesgo en el ámbito internacional y nacional al cual Colombia se acogió.

A nivel internacional el incremento en las preocupaciones sobre los efectos de los desastres naturales en los Derechos Humanos desencadenaron en el año 1994 la celebración de la Conferencia Mundial sobre reducción de desastres naturales, en la cual se adoptaron los principios, estrategia y plan de acción de Yokohama. Ese mismo año se aprobó el Programa de Acción para el desarrollo sostenible de los pequeños Estados insulares en desarrollo, figuras que se constituyen en el antecedente directo de la Conferencia Mundial sobre reducción de los desastres, de Hyogo, en donde se adopta la Estrategia 2005 - 2015: Aumento de la Resiliencia de las naciones y las comunidades ante los desastres, materia que igualmente recibió destacado tratamiento en la Cumbre Mundial sobre Desarrollo Sostenible de Johannesburgo en 2002, y la Conferencia Internacional sobre sistemas de alerta temprana celebrada en 
Bonn (Alemania) en 2003. En el año 2005 fue aprobada la Estrategia de Mauricio para la ejecución ulterior del Programa de Acción para el desarrollo sostenible de los pequeños Estados Insulares en desarrollo, para concluir con el Marco de Acción de Sendai (MAS) para la Reducción del Riesgo de Desastres, vigente entre el periodo $2015-2030$.

La correlación entre la gestión del riesgo de desastres y el ordenamiento territorial en Colombia resulta incidida con fuerza a partir de la Declaración de Rio de Janeiro de 1992, de Yokohama 1994 y de Hyogo 2005, pero no sucedió igual con el reasentamiento y reubicación figuras que reciben impulso desde Hyogo y en la actualidad desde algunas directrices del MAS, sin que sea posible asegurar que los tres temas tengan el mismo nivel de desarrollo y jerarquía en el ámbito internacional, y menos en el orden interno. La tendencia internacional motivó el desarrollo normativo e institucional en Colombia, donde la correlación entre gestión del riesgo de desastres y el ordenamiento territorial se formaliza en la década de los noventa alcanzando una articulación nominal que no es armónica, aspecto que solo hasta los inicios de la segunda década del siglo XXI trata de ser equilibrada, y a la cual le queda pendiente la compenetración con el reasentamiento y la reubicación poblacional, tal como se evidencia en la evolución de las normas nacionales.

En el periodo en el que la gestión del riesgo de desastres se desarrolló estrechamente bajo el amparo del goce a un ambiente sano, en el Decreto 1355 de 1970, anterior Código Nacional de Policía, las competencias para la gestión del riesgo fueron concentradas de manera predominante en las autoridades del orden municipal de naturaleza policiva, sin que se estructurara un sistema a nivel nacional y en coordinación con otras circunscripciones. De lo anterior que el Decreto 1355 de 1970 no se expidiera como una norma exclusivamente destinada a la regulación de la gestión del riesgo, sino que planteaba de manera aislada preceptos que otorgaban funciones de manejo ambiental y de calamidades públicas a ciertas autoridades con la única finalidad de establecer procedimientos y competencias para hacerle frente a los desastres ambientales y de salubridad pública que se pudieran presentar. Por lo anterior, el ejercicio de los Derechos Humanos se enmarcaba en un escenario reactivo, policivo y asistemático en el cual el orden municipal no evidenciaba un referente jurídico expreso que impusiera la obligación de correlacionar las figuras.

Con la Ley 9 de 1979 la situación tiene leves avances, en la medida que desde el subtema de "planeamiento de las operaciones de emergencia", el artículo 499 disponía la obligación de todas las circunscripciones de participar en el planeamiento de las operaciones de emergencia en sus respectivas comunidades, incluyendo todas aquellas que podían albergar grupos de personas en caso de emergencia. El artículo 500 dispuso el tipo de desastres, las autoridades responsables, sus funciones, y los lugares donde se ubicaría a la comunidad afectada tanto en tiempo de desastre como en su vida cotidiana, así como los elementos mínimos que debía tener el planeamiento de operaciones de emergencias; y bajo el título de "planes de contingencia" el artículo 501 señaló que cada comité de contingencia debía elaborar un plan para su respectiva jurisdicción de acuerdo al análisis de vulnerabilidad, donde se incluía el tipo de desastre que podía presentarse según la región. Esta normativa acogió la dimensión planeadora de la capacidad de respuesta frente al evento, pero descuido el potencial preventivo de la planeación a través del ordenamiento territorial.

Solo con la Ley 46 de 1988 se creó y organizó el Sistema Nacional para la prevención y atención de desastres (SINPAD), hoy derogado por la Ley 1523 de 2012. Esa normativa no solo trazó como objetivos definir las responsabilidades y funciones de todos los organismos y entidades públicas, privadas y comunitarias, en las fases de prevención, manejo, rehabilitación, reconstrucción y desarrollo a que dan lugar las situaciones de desastre; integrar los esfuerzos públicos y privados para la adecuada prevención y atención de las situaciones de desastre; y garantizar un manejo oportuno y eficiente de todos los recursos humanos, técnicos, administrativos y económicos que sean indispensables para la prevención y atención del mismo, sino que encargó a la Oficina Nacional para la Atención de Desastres de elaborar el Plan Nacional para la Prevención y Atención de Desastres, que desde el artículo 5 debía ser tenido en cuenta como parámetro por los organismos de planeación de orden regional, departamental y municipal en sus respectivas elaboraciones. 
Esta Ley permite afirmar que la integración expresa entre la gestión del riesgo de desastres y la incidencia en la planeación y el ordenamiento territorial de las circunscripciones tuvo lugar a la luz del SINPAD. Se enfocó con preferencia en la organización administrativa territorial que luego sería perfeccionada con la descentralización administrativa, promovió un funcionamiento sistémico guiado por la coordinación, la concurrencia, la subsidiariedad y la complemetariedad como principios administrativos, y finalmente, generó un antecedente vinculante para las entidades de menor jerarquía sin hacer trasgresión a la autonomía que las mismas ya habían recibido para la época a través de normas como el Decreto Ley 1222 y 1333 de 1986, tal como se replica en la actualidad desde distintas normas nacionales. Sin embargo, las preocupaciones por los procesos de reasentamiento y reubicación poblacional resultantes de riesgos de desastre o catástrofes en la planeación del territorio pasaron de largo en la consolidación del sistema, y con ello, la posibilidad de adoptar e implementar mecanismos formales que guiaran el ejercicio de la función administrativa de las autoridades competentes para la protección de Derechos Humanos.

La gestión del riesgo de desastres adquiere con el SINPAD el punto cumbre de desarrollo a través de la reglamentación del decreto 919 de 1989, norma que mantuvo su vigencia hasta el año 2012. La estructura administrativa sufrió una ampliación que no se había registrado en la institucionalidad nacional, toda vez que el SINPAD estaría conformado por el Comité Nacional para la Prevención y Atención de Desastres, los comités regionales y locales para la prevención y atención de desastres, la oficina nacional para la atención de desastres, el comité técnico nacional para la prevención y atención de desastres, el comité operativo nacional para atención de desastres, los ministerios y departamentos administrativos, las entidades descentralizadas del orden nacional y las entidades territoriales y sus entidades descentralizadas en cuanto sus competencias y funciones tuvieran relación con las actividades de prevención y atención de desastres, la sociedad nacional de la cruz roja colombiana y las entidades y personas privadas que por su objeto y funciones tuvieran relación con dichas actividades. Desde esta nueva e integral normativa, la correlación entre la gestión del riesgo de desastres y el ordenamiento territorial marcaría un fuerte paso de formalización y articulación, por lo menos de carácter dispositivo, correlación que sería objeto de desarrollos progresivos en normas predecesoras. Al mismo tiempo deja los cimientos de la correlación con el reasentamiento poblacional, tema residual que a diferencia del ordenamiento territorial, se mantuvo secular en el orden jurídico e institucional, incluso hasta nuestros días.

Inicialmente en el Decreto 919 de 1989 sobresalía la obligación de los moradores de permitir la ocupación de inmuebles en caso de desastre, por el tiempo estrictamente necesario y previa autorización de la Oficina Nacional de Atención de Desastres o del presidente del comité regional o local, por parte de cualquier entidad pública y en virtud de la función social de la propiedad, precepto que era aplicable no solo a los propietarios sino también a los poseedores y tenedores de inmuebles, predios y mejoras. De otra parte regulaba la orden de demolición por deterioro del bien inmueble que podría ser decretada por el alcalde del municipio en virtud de una declaratoria de situación de desastre cuando el deterioro del bien pusiera en peligro la seguridad de los habitantes del inmueble o de las demás personas; y regulaba los estudios sobre la localización de asentamientos humanos y edificaciones, preceptuando que la Oficina Nacional para la Atención de Desastres o los Comités Regionales y Locales debían promover estudios que permitieran determinar en qué áreas de influencias de la declaratoria de situación de desastres no podrían ubicarse asentamientos humanos ni construir edificaciones por razones ambientales de peligro o riesgos. Así pues, basado en los mencionados estudios el alcalde debía ordenar la reubicación de las poblaciones implicadas dentro de plazos prudenciales ordenando también las demoliciones necesarias. En la sección IV de la norma, titulada "adquisición y expropiación" señalaba en su artículo 37 las entidades autorizadas para adquirir o expropiar bienes inmuebles en virtud de la declaratoria de situación de desastres, el artículo 38 reguló la negoción directa, el artículo 39 la expropiación y el 40 la declaratoria de utilidad pública e interés social para efectos de llevar a cabo dicho procedimiento, figuras que en términos de otras normas, subsisten en su finalidad.

Se lee en los artículos precedentes la supremacía del interés general (concebido en este caso desde la necesidad de prevenir y atender las situaciones de desastres y de la función social de la propiedad privada), respecto a los derechos patrimoniales de carácter individual, sin detrimento de la garantía del 
derecho al debido proceso y del acceso a la administración de justicia que poseen los titulares de derechos sobre los inmuebles a afectar.

Las garantías jurídicas para ejercer los Derechos Humanos se verían también reforzadas en la correlación entre la gestión del riesgo de desastres y el ordenamiento territorial a través de una ley de significativo valor en materia de ordenamiento territorial urbano: La Ley 9 de 1989. En ella sobresale una visión articulada de la gestión del riesgo y el ordenamiento territorial a la sombra de la autonomía territorial, cuando a los planes de desarrollo se les impuso como uno de los componentes la reserva de tierras urbanizables para atender oportuna y adecuadamente la demanda por vivienda de interés social y para reubicar aquellos asentamientos humanos que presentan graves riesgos para la salud e integridad personal de sus habitantes (Articulo 2), contempló como motivo de utilidad pública para emprender expropiaciones la "Reubicación de asentamientos humanos ubicados en sectores de alto riesgo y rehabilitación de inquilinatos" (Artículo 10), pero sobre todo, a partir de la disposición del artículo 56, aún vigente, por medio del cual a las autoridades del orden municipal se les impone:

(...) levantar, en el término máximo de seis (6) meses contados a partir de la vigencia de la presente Ley, un inventario de los asentamientos humanos que presenten altos riesgos para sus habitantes, en razón a su ubicación en sitios anegadizos, o sujetos a derrumbes y deslizamientos, o que de otra forma presenten condiciones insalubres para la vivienda y reubicarán a estos habitantes en zonas apropiadas, con la participación del Instituto de Crédito Territorial. Además, tomarán todas las medidas y precauciones necesarias para que el inmueble desocupado no vuelva a ser usado para vivienda humana.

Para ello la norma habilita la posibilidad de adquirir los predios de las personas a ser reubicadas a través de enajenación voluntaria o expropiación, y faculta, en el mismo sentido policivo de la década de los años setenta, al Alcalde y autoridades de policía a "ordenar la desocupación (...), y la demolición de las edificaciones afectadas. Esta orden se considerará, para todos los efectos, como una orden policiva en los términos del Código Nacional de Policía." Esta disposición, que articulada a otras provenientes de la Ley 388 de 1997 (Artículos 8 y 13) y la Ley 715 de 2001 (Articulo 76), a criterio de la Corte Constitucional de Colombia llegan a definir el sendero de la política pública de gestión del riesgo de desastres (Sentencia T - 041, T - 106 y T - 865 de 2011). Sin embargo en este entramado jurídico institucional de la gestión del riesgo de desastres y ordenamiento territorial, en donde el reasentamiento y la reubicación encuentran un lugar aunque escaso, visible ¿Cómo se comprendió la figura del asentamiento? ¿Cómo la de reasentamiento? ¿Qué diferencia se estableció entre reasentamiento y reubicación? Pero más allá, ¿Cómo quedó definido para el ordenamiento territorial desde la gestión del riesgo el procedimiento a seguir con las poblaciones propensas a procesos de reasentamiento? o ¿Acaso fue el ordenamiento territorial el que proporcionó la solución del vacío jurídico institucional?

La promulgación de la Ley 388 de 1997 implicó el fortalecimiento de la correlación entre el ordenamiento territorial y la gestión del riesgo de desastres a partir de la protocolización de dicha materia como una expresión especializada del sector ambiental, el cual, desde entonces, es criterio imperativo para el ordenamiento territorial. La Ley 388 señala como uno de sus objetivos (Artículo 1, numerales 2 y 3 ) el establecimiento de mecanismos que permitan al municipio la prevención de desastres en asentamientos de alto riesgo. Además la norma establece que el ordenamiento territorial es una función pública que pretende alcanzar, entre otros, una mayor seguridad de los asentamientos humanos ante los riesgos naturales (artículo 3, numeral 4). Fija como modalidades de acciones urbanísticas la determinación de las zonas no urbanizables que presenten riesgos para la localización de asentamientos humanos, por amenazas naturales, o que de otra forma presenten condiciones insalubres para la vivienda (artículo 8, numeral 5) y la localización de las áreas críticas de recuperación y control para la prevención de desastres, así como las áreas con fines de conservación y recuperación paisajística (artículo 8, numeral 11).

En el artículo 10 señala entre otros, que la prevención de amenazas y riesgos naturales constituyen uno de los elementos determinantes que se deberán tener en cuenta para la elaboración y adopción de los Planes de Ordenamiento Territorial (POT), el cual deberá contener dentro de su componente general la determinación y ubicación en planos de las zonas que presenten alto riesgo para la localización de asentamientos humanos, por amenazas, riesgos naturales o condiciones de insalubridad (artículo 12 , 
numeral 2.3). Además los POT dentro de su componente urbano deberán tener en cuenta la delimitación de áreas expuestas a amenazas y riesgos naturales y el establecimiento de mecanismos para la reubicación de los asentamientos humanos localizados en zonas de alto riesgo donde se incluyan estrategias para evitar su nueva ocupación. (Artículo 13, numerales 3 y 5). El componente rural del POT debe establecer como mínimo, entre otros, la delimitación de las áreas de conservación y protección de los recursos naturales paisajísticos, geográficos y ambientales, incluyendo las áreas de amenazas y riesgos, o que formen parte de los sistemas de provisión de los servicios públicos domiciliarios o de disposición final de desechos sólidos o líquidos (Artículo 14, numeral 3) contenidos que deberán ser tenidos en cuenta también en los planes básicos de ordenamiento territorial en cada uno de sus componentes según lo preceptuado en el artículo 16 de la Ley 388 , y por mandato del artículo 17, los esquemas de ordenamiento territorial, que deberán contener como mínimo los objetivos, estrategias y políticas a largo y mediano plazo para la determinación de las zonas de amenazas y riesgos naturales y las medidas de protección.

Como justificación de utilidad pública para motivar acto administrativo expropiatorio, el artículo 58 (literales b y $\mathrm{m}$ ) establece la reubicación de asentamientos humanos ubicados en sectores de alto riesgo y el traslado de las poblaciones por riesgos físicos inminentes, $y$, adicionalmente establece sanciones para quienes parcelen, urbanicen o construyan en zonas calificadas como de riesgo. (Artículo 104, numeral 1). Finalmente, la Ley 388 prevé en el artículo 35 la categorización de suelo de protección para las áreas de amenazas y riesgos no mitigable donde existan asentamientos humanos, las cuales tienen restringida la posibilidad de urbanizarse; además, como tratamiento de las áreas catalogadas como de riesgo no recuperable que hayan sido desalojadas, prevé la entrega de las mismas a las Corporaciones Autónomas Regionales o la autoridad ambiental de la respectiva circunscripción territorial para su manejo y evitación de una nueva ocupación. Dicho desalojo deberá efectuarse mediante planes o proyectos de reubicación de asentamientos humanos (Artículo 121), planes o proyectos que a la fecha, se mantienen al amparo de la autonomía de las entidades territoriales, específicamente de las municipales, las cuales recibe de la Ley 1454 de 2011 la cláusula residual de competencias en materia de ordenamiento territorial.

Para finales de la década de los años noventa las premisas propuestas arriba han cumplido su curso: la gestión del riesgo de desastres, el ordenamiento territorial, y el reasentamiento poblacional son materias que dentro de la evolución jurídico institucional colombiana no surgieron articuladas, por el contrario, su progresiva evolución nacional e internacional fue la que impulsó la correlación entre las mismas. Dicha correlación cuenta con un respaldo normativo e institucional desequilibrado, poco armónico e incompleto, el cual, después del amplio desarrollo jurídico continua privilegiando los esfuerzos jurídicos, políticos e institucionales por el goce a un ambiente sano en el cual no se ha dado el lugar debido a la seguridad jurídica de los procesos de reasentamiento y reubicación poblacional como componente tanto del ambiente sano como de la gestión del riesgo en sus dimensiones de derechos colectivos constitucional y legalmente reconocidos en Colombia.

Y ante la histórica necesidad de promulgar una legislación garantista para el ejercicio de los Derechos Humanos que regule las generalidades de los procesos de reasentamiento y reubicación de población, la normativa reciente vigente tampoco logra llenar el vacío del reasentamiento y la reubicación poblacional, dejando desprovisto este espectro del ejercicio de los Derechos Humanos. La Ley 1454 de 2011, orgánica de ordenamiento territorial, solo le deja a los departamentos la función de "Definir las políticas de asentamientos poblacionales y centros urbanos, de tal manera que facilite el desarrollo de su territorio." (Artículo 29, b) la Ley 1753 de 2015 sobre el Plan Nacional de Desarrollo 2014 - 2018, bajo la denominación de reasentamiento, dispone que las entidades públicas deben ejecutar dichos procesos, de manera conjunta conforme a las competencias y funciones, pero, sujetas a "las condiciones que señale el reglamento", reglamento que si es nacional, aún no ha sido adoptado, y si se deja a la autonomía de las entidades territoriales, profundiza la aparición de procedimientos diversos y disimiles en los territorios municipales del país sin definir un marco de seguridad jurídica para el efectivo ejercicio de los Derechos Humanos. Pero más allá de estas normas, la Ley 1523 de 2012, en sus esfuerzos por incorporar la gestión del riesgo en la inversión pública (Artículo 38), a la planificación territorial y del desarrollo (Artículo 39), a la planificación (Artículo 40), de reforzar la articulación con el ordenamiento 
territorial y la planeación del desarrollo (Artículo 41) tampoco logró regular condiciones diferentes a las ya existentes sobre el reasentamiento y reubicación poblacional, condiciones que se caracterizan por la amplitud y vaguedad de una acción político administrativa que se mantiene a la sombra de un procedimiento desregularizado.

\section{UN CONTRASTE ENTRE LA EVOLUCIÓN JURIDICO INSTITUCIONAL Y EL ACUMULADO HISTÓRICO DERIVADO DE LOS EVENTOS NATURALES CATASTRÓFICOS}

La evolución del marco normativo e institucional para la gestión del riesgo de desastres en Colombia queda sujeto a una potencial ineficacia si se tienen presentes los resultados de la sistematización y consecuente consolidación del número de muertos, heridos, desparecidos, personas, y familias registradas a causa de avalanchas, contaminación, deslizamiento, erosión, granizadas, heladas, inundaciones, marejadas, sequías, sismos, tormentas eléctricas, vendavales y erupciones volcánicas registradas ante la UNGRD en los treinta y dos departamentos y el Distrito Capital en el periodo 2000 2014. Desde las cifras se pone de presente la existencia de una evidente falla de la gestión del riesgo a partir de los potenciales de previsibilidad, pues por ejemplo, el número de repeticiones de diversos eventos naturales en el período que comprende los años 2007 a 2012 aumenta tendencialmente y no presenta variaciones significativas de disminución, lo que hace de los eventos procesos que pudieron ser prevenidos y mitigados desde el factor de previsibilidad y análisis de vulnerabilidad por lo menos entorno a sus consecuencias si se tiene en cuenta su fuerte y común presencia en el periodo histórico previo al año 2000 y posterior al año 2012. Lo anterior se puede ejemplificar a partir de los dos de los eventos naturales de mayor frecuencia en el territorio nacional: Inundación y deslizamiento.

\begin{tabular}{|c|c|c|c|c|c|c|c|c|c|c|c|c|c|c|c|}
\hline \multicolumn{16}{|c|}{ FRECUENCIA DE EVENTO NATURAL INUNDACIÓN DISCRIMINADO POR DEPARTAMENTOS ENTRE 2000 - 2014} \\
\hline DEPARTAMENTO & 2000 & 2001 & 2002 & 2003 & 2004 & 2005 & 2006 & 2007 & 2008 & 2009 & 2010 & 2011 & 2012 & 2013 & 2014 \\
\hline Amazonas & 8 & 2 & 8 & 3 & 2 & 0 & 1 & 1 & 2 & 5 & 1 & 3 & 2 & 0 & 1 \\
\hline Antioquia & 23 & 11 & 10 & 20 & 19 & 43 & 36 & 46 & 95 & 62 & 118 & 160 & 56 & 58 & 27 \\
\hline Arauca & 4 & 1 & 6 & 5 & 7 & 2 & 19 & 5 & 6 & 11 & 9 & 15 & 24 & 9 & 8 \\
\hline Atlántico & 10 & 3 & 5 & 7 & 19 & 27 & 14 & 40 & 46 & 10 & 54 & 56 & 37 & 22 & 6 \\
\hline Bolívar & 1 & 1 & 15 & 26 & 55 & 44 & 6 & 66 & 78 & 6 & 67 & 72 & 23 & 12 & 15 \\
\hline Boyacá & 2 & 0 & 4 & 15 & 9 & 10 & 36 & 5 & 43 & 11 & 48 & 3 & 82 & 18 & 8 \\
\hline Caldas & 1 & 0 & 1 & 2 & 5 & 6 & 4 & 5 & 13 & 0 & 13 & 22 & 27 & 12 & 6 \\
\hline Caquetá & 4 & 3 & 1 & 2 & 5 & 3 & 1 & 8 & 2 & 9 & 10 & 21 & 17 & 18 & 15 \\
\hline Casanare & 3 & 0 & 17 & 11 & 13 & 22 & 22 & 5 & 16 & 12 & 26 & 23 & 46 & 18 & 11 \\
\hline Cauca & 5 & 1 & 7 & 2 & 16 & 12 & 14 & 13 & 81 & 24 & 35 & 74 & 55 & 22 & 29 \\
\hline Cesar & 32 & 6 & 2 & 8 & 19 & 20 & 20 & 45 & 42 & 12 & 48 & 62 & 32 & 17 & 13 \\
\hline Chocó & 18 & 15 & 27 & 35 & 15 & 36 & 8 & 29 & 39 & 24 & 46 & 71 & 68 & 38 & 24 \\
\hline Córdoba & 3 & 4 & 3 & 25 & 31 & 9 & 21 & 42 & 39 & 26 & 45 & 42 & 50 & 22 & 11 \\
\hline Cundinamarca & 4 & 4 & 11 & 10 & 17 & 23 & 59 & 18 & 109 & 33 & 85 & 242 & 122 & 49 & 36 \\
\hline Guainía & 0 & 0 & 1 & 1 & 1 & 26 & 1 & 1 & 0 & 0 & 0 & 1 & 1 & 1 & 1 \\
\hline Guaviare & 1 & 1 & 1 & 2 & 1 & 0 & 0 & 2 & 2 & 0 & 0 & 4 & 1 & 2 & 1 \\
\hline Huila & 12 & 0 & 4 & 0 & 10 & 26 & 3 & 12 & 46 & 19 & 33 & 82 & 68 & 30 & 18 \\
\hline Guajira & 0 & 2 & 5 & 3 & 14 & 22 & 6 & 17 & 27 & 8 & 27 & 24 & 15 & 10 & 4 \\
\hline Magdalena & 18 & 3 & 4 & 9 & 32 & 36 & 32 & 58 & 44 & 8 & 58 & 65 & 34 & 16 & 11 \\
\hline Meta & 15 & 0 & 15 & 2 & 13 & 16 & 15 & 18 & 24 & 12 & 37 & 42 & 10 & 14 & 29 \\
\hline Nariño & 22 & 0 & 7 & 1 & 21 & 11 & 6 & 13 & 30 & 17 & 67 & 37 & 68 & 30 & 33 \\
\hline Norte de Santander & 10 & 2 & 15 & 15 & 23 & 35 & 11 & 2 & 32 & 12 & 34 & 70 & 28 & 0 & 7 \\
\hline Putumayo & 12 & 7 & 0 & 2 & 5 & 2 & 3 & 6 & 6 & 11 & 5 & 4 & 47 & 38 & 23 \\
\hline Quindío & 2 & 2 & 0 & 2 & 2 & 4 & 16 & 3 & 13 & 6 & 15 & 43 & 15 & 27 & 5 \\
\hline Risaralda & 2 & 6 & 6 & 1 & 2 & 19 & 12 & 15 & 12 & 6 & 22 & 44 & 31 & 12 & 8 \\
\hline San Andrés y Prov. & 0 & 0 & 0 & 1 & 0 & 0 & 1 & 0 & 0 & 0 & 0 & 0 & 2 & 0 & 1 \\
\hline Santander & 19 & 10 & 20 & 33 & 21 & 44 & 49 & 29 & 55 & 47 & 56 & 90 & 19 & 28 & 21 \\
\hline Sucre & 13 & 2 & 6 & 11 & 17 & 15 & 19 & 28 & 23 & 7 & 28 & 36 & 36 & 25 & 12 \\
\hline Tolima & 19 & 11 & 14 & 9 & 13 & 30 & 16 & 26 & 83 & 17 & 61 & 55 & 60 & 62 & 19 \\
\hline Valle del Cauca & 27 & 7 & 6 & 8 & 4 & 21 & 37 & 54 & 75 & 24 & 67 & 122 & 78 & 59 & 26 \\
\hline Vaupés & 0 & 0 & 1 & 0 & 0 & 1 & 0 & 0 & 0 & 0 & 0 & 1 & 0 & 3 & 2 \\
\hline Vichada & 1 & 0 & 2 & 4 & 4 & 0 & 2 & 4 & 3 & 0 & 0 & 1 & 4 & 1 & 4 \\
\hline TOTAL ANUAL & 291 & 104 & 224 & 275 & 415 & 565 & 490 & 616 & 1086 & 439 & 1115 & 1587 & 1158 & 673 & 435 \\
\hline
\end{tabular}




\begin{tabular}{|c|c|c|c|c|c|c|c|c|c|c|c|c|c|c|c|}
\hline \multicolumn{16}{|c|}{ FRECUENCIA DE EVENTO NATURAL DESLIZAMIENTO DISCRIMINADO POR DEPARTAMENTOS ENTRE 2000 - 2014} \\
\hline DEPARTAMENTO & 2000 & 2001 & 2002 & 2003 & 2004 & 2005 & 2006 & 2007 & 2008 & 2009 & 2010 & 2011 & 2012 & 2013 & 2014 \\
\hline Amazonas & 0 & 0 & 0 & 0 & 0 & 0 & 0 & 0 & 2 & 0 & 0 & 1 & 0 & 0 & 0 \\
\hline Antioquia & 16 & 6 & 14 & 4 & 14 & 21 & 28 & 14 & 43 & 22 & 39 & 44 & 26 & 38 & 19 \\
\hline Arauca & 0 & 0 & 0 & 0 & 0 & 0 & 0 & 0 & 0 & 0 & 0 & 1 & 0 & 0 & 0 \\
\hline Atlántico & 0 & 0 & 0 & 0 & 0 & 1 & 2 & 0 & 1 & 0 & 17 & 10 & 1 & 1 & 1 \\
\hline Bolívar & 1 & 0 & 0 & 2 & 1 & 1 & 3 & 3 & 2 & 1 & 6 & 8 & 8 & 20 & 7 \\
\hline Boyacá & 7 & 3 & 21 & 4 & 11 & 8 & 11 & 2 & 20 & 5 & 58 & 101 & 43 & 20 & 5 \\
\hline Caldas & 8 & 1 & 2 & 4 & 5 & 23 & 13 & 21 & 33 & 7 & 23 & 3 & 9 & 18 & 7 \\
\hline Caquetá & 0 & 0 & 0 & 1 & 1 & 1 & 0 & 2 & 0 & 1 & 2 & 5 & 5 & 6 & 7 \\
\hline Casanare & 0 & 0 & 0 & 0 & 0 & 0 & 0 & 0 & 1 & 0 & 0 & 5 & 5 & 3 & 5 \\
\hline Cauca & 20 & 3 & 0 & 2 & 3 & 9 & 5 & 17 & 36 & 5 & 35 & 39 & 25 & 19 & 7 \\
\hline Cesar & 2 & 1 & 0 & 0 & 0 & 0 & 1 & 0 & 3 & 1 & 4 & 4 & 5 & 1 & 3 \\
\hline Chocó & 0 & 0 & 0 & 2 & 1 & 5 & 5 & 3 & 11 & 11 & 8 & 13 & 3 & 7 & 12 \\
\hline Córdoba & 0 & 0 & 0 & 1 & 0 & 0 & 0 & 2 & 0 & 0 & 0 & 0 & 1 & 3 & 1 \\
\hline Cundinamarca & 1 & 0 & 3 & 4 & 8 & 9 & 31 & 10 & 32 & 19 & 60 & 93 & 48 & 41 & 44 \\
\hline Guainía & 0 & 0 & 0 & 0 & 0 & 0 & 0 & 0 & 0 & 10 & 0 & 0 & 0 & 1 & 0 \\
\hline Guaviare & 0 & 0 & 0 & 0 & 0 & 0 & 0 & 0 & 0 & 1 & 0 & 0 & 0 & 0 & 1 \\
\hline Huila & 3 & 1 & 1 & 2 & 5 & 7 & 0 & 0 & 0 & 12 & 22 & 88 & 59 & 24 & 2 \\
\hline Guajira & 0 & 0 & 0 & 0 & 0 & 0 & 3 & 5 & 6 & 0 & 1 & 1 & 0 & 1 & 0 \\
\hline Magdalena & 2 & 0 & 0 & 0 & 1 & 1 & 1 & 0 & 0 & 0 & 2 & 0 & 0 & 0 & 1 \\
\hline Meta & 0 & 0 & 1 & 0 & 2 & 1 & 4 & 1 & 3 & 1 & 7 & 6 & 4 & 0 & 1 \\
\hline Nariño & 29 & 0 & 1 & 0 & 4 & 11 & 38 & 13 & 34 & 38 & 63 & 132 & 125 & 51 & 28 \\
\hline Norte de Santander & 7 & 2 & 1 & 8 & 7 & 10 & 16 & 3 & 12 & 10 & 13 & 37 & 30 & 16 & 13 \\
\hline Putumayo & 0 & 0 & 0 & 0 & 1 & 0 & 19 & 1 & 2 & 1 & 3 & 2 & 4 & 5 & 4 \\
\hline Quindío & 2 & 0 & 0 & 2 & 4 & 5 & 0 & 4 & 4 & 3 & 6 & 44 & 13 & 23 & 22 \\
\hline Risaralda & 3 & 1 & 2 & 4 & 4 & 16 & 11 & 13 & 8 & 5 & 21 & 94 & 32 & 28 & 20 \\
\hline San Andrés y Prov. & 0 & 0 & 0 & 0 & 0 & 0 & 0 & 0 & 0 & 0 & 0 & 0 & 0 & 0 & 0 \\
\hline Santander & 9 & 1 & 3 & 2 & 16 & 12 & 6 & 6 & 5 & 6 & 15 & 5 & 63 & 50 & 40 \\
\hline Sucre & 0 & 0 & 0 & 0 & 0 & 0 & 0 & 1 & 1 & 0 & 0 & 1 & 0 & 0 & 0 \\
\hline Tolima & 12 & 4 & 8 & 9 & 3 & 11 & 11 & 9 & 16 & 10 & 27 & 29 & 18 & 46 & 44 \\
\hline Valle del Cauca & 8 & 3 & 0 & 2 & 3 & 8 & 23 & 6 & 20 & 6 & 23 & 26 & 13 & 21 & 11 \\
\hline Vaupés & 0 & 0 & 0 & 0 & 0 & 0 & 0 & 0 & 0 & 0 & 0 & 0 & 0 & 0 & 0 \\
\hline Vichada & 0 & 0 & 0 & 0 & 0 & 0 & 0 & 0 & 0 & 0 & 0 & 1 & 0 & 0 & 0 \\
\hline TOTAL ANUAL & 130 & 26 & 57 & 53 & 94 & 160 & 231 & 136 & 295 & 175 & 455 & 793 & 540 & \begin{tabular}{|l|}
443 \\
\end{tabular} & 305 \\
\hline
\end{tabular}

Los consolidados nacionales dan cuenta que los eventos con mayor frecuencia en el territorio son los hidrometeorológicos (como avalancha, deslizamiento e inundación) que al tiempo se constituyen como los que más afectan el territorio nacional, son los más desastrosos en cuanto a la afectación a población en condiciones de vulnerabilidad en contravía con otros eventos atendidos por la institucionalidad y la sociedad como el sismo y la erupción. Algunos picos de incrementos desmedidos de víctimas permiten sostener las anteriores apreciaciones. Al igual que se ejemplifica con los más de 300 muertos de la reciente avenida torrencial de Mocoa en abril de 2017 se puede hacer con las cifras del período de 20102011, años durante los cuales se da la denominada "Ola Invernal", y frente a la cual el Estado, antes que prevenir o hacer esfuerzos para mitigar, terminó reaccionando frente a la catástrofe, a pesar de su previsibilidad por la dinámica de sus condiciones en años anteriores tales como 2006 y 2007.

\begin{tabular}{|l|c|c|c|c|c|c|c|c|c|c|c|c|c|c|c|}
\hline \multicolumn{1}{|c|}{ TOTAL NACIONAL DE NÚMERO DE MUERTOS POR EVENTOS HIDROMETEOROLÓGICOS 2000 - 2014 } \\
\hline \multicolumn{1}{|c|}{ FENÓNEMO } & $\mathbf{2 0 0 0}$ & $\mathbf{2 0 0 1}$ & $\mathbf{2 0 0 2}$ & $\mathbf{2 0 0 3}$ & $\mathbf{2 0 0 4}$ & $\mathbf{2 0 0 5}$ & $\mathbf{2 0 0 6}$ & $\mathbf{2 0 0 7}$ & $\mathbf{2 0 0 8}$ & $\mathbf{2 0 0 9}$ & $\mathbf{2 0 1 0}$ & $\mathbf{2 0 1 1}$ & $\mathbf{2 0 1 2}$ & $\mathbf{2 0 1 3}$ & $\mathbf{2 0 1 4}$ \\
\hline AVALANCHA & 0 & 0 & 7 & 11 & 0 & 57 & 12 & 10 & 15 & 10 & 25 & 8004 & 261 & 0 & 7 \\
\hline DESLIZAMIENTO & 48 & 57 & 20 & 49 & 41 & 90 & 129 & 59 & 140 & 47 & 141 & 145 & 56 & 36 & 33 \\
\hline INUNDACIÓN & 29 & 6 & 12 & 42 & 13 & 76 & 18 & 32 & 24 & 34 & 72 & 118 & 22 & 19 & 13 \\
\hline $\begin{array}{l}\text { TORMENTA } \\
\text { ELÉCTRICA }\end{array}$ & 0 & 0 & 0 & 0 & 0 & 0 & 8 & 1 & 1 & 2 & 14 & 14 & 23 & 18 & 0 \\
\hline VENDAVAL & 0 & 2 & 4 & 1 & 2 & 65 & 8 & 6 & 4 & 1 & 5 & 9 & 6 & 4 & 97 \\
\hline TOTAL ANUAL & $\mathbf{7 7}$ & $\mathbf{6 5}$ & $\mathbf{4 3}$ & $\mathbf{1 0 3}$ & $\mathbf{5 6}$ & $\mathbf{2 8 8}$ & $\mathbf{1 7 5}$ & $\mathbf{1 0 8}$ & $\mathbf{1 8 4}$ & $\mathbf{9 4}$ & $\mathbf{2 5 7}$ & $\mathbf{8 2 9 0}$ & $\mathbf{3 6 8}$ & $\mathbf{7 7}$ & $\mathbf{1 5 0}$ \\
\hline
\end{tabular}


TOTAL NACIONAL DE HERIDOS POR EVENTOS HIDROMETEOROLÓGICOS 2000 - 2014 \begin{tabular}{|l|c|c|c|c|c|c|c|c|c|c|c|c|c|c|}
\hline FENÓNEMO & 2000 & 2001 & 2002 & 2003 & 2004 & 2005 & 2006 & 2007 & 2008 & 2009 & 2010 & 2011 & 2012 & 2013 \\
2014 \\
\hline
\end{tabular}

\begin{tabular}{|c|c|c|c|c|c|c|c|c|c|c|c|c|c|c|c|}
\hline AVALANCHA & 0 & 0 & 0 & 15 & 7 & 80 & 10 & 1 & 6 & 15 & 5 & 8003 & 194 & 1 & 1 \\
\hline DESLIZAMIENTO & 71 & 34 & 16 & 65 & 70 & 101 & 129 & 76 & 170 & 34 & 182 & 150 & 72 & 87 & 17 \\
\hline $\begin{array}{l}\text { TORMENTA } \\
\text { ELÉCTRICA }\end{array}$ & 0 & 0 & 0 & 0 & 0 & 0 & 66 & 5 & 0 & 3 & 67 & 0 & 33 & 31 & 0 \\
\hline VENDAVAL & 18 & 225 & 3 & 5 & 16 & 0 & 112 & 28 & 34 & 31 & 30 & 13 & 194 & 52 & 374 \\
\hline TOTAL ANUAL & 89 & 259 & 19 & 85 & 93 & 181 & 317 & 110 & 210 & 83 & 284 & 8166 & 493 & 171 & 392 \\
\hline
\end{tabular}

\begin{tabular}{|c|c|c|c|c|c|c|c|c|c|c|c|c|c|}
\hline \multicolumn{14}{|c|}{ TOTAL NACIONAL DE FAMILIAS REGISTRADAS POR EVENTOS HIDROMETEOROLÓGICOS 2000 - 2012} \\
\hline FENÓNEMO & 2000 & 2001 & 2002 & 2003 & 2004 & 2005 & 2006 & 2007 & 2008 & 2009 & 2010 & 2011 & 2012 \\
\hline AVALANCHA & 437 & 21 & 1165 & 583 & 2427 & 4858 & 2158 & 830 & 2665 & 1503 & 23241 & 8006 & 3154 \\
\hline CONTAMINACIÓN & 1204 & 0 & 0 & 5 & 0 & 0 & 641 & 9 & 0 & 4 & 0 & 0 & 0 \\
\hline DESLIZAMIENTO & 5598 & 810 & 3427 & 1394 & 8479 & 11620 & 9732 & 3873 & 14661 & 8378 & 18949 & 9 & 11527 \\
\hline EROSIÓN & 0 & 0 & 0 & 0 & 583 & 294 & 0 & 1557 & 0 & 154 & 691 & 0 & 77 \\
\hline ERUPCIÓN & 0 & 0 & 0 & 30 & 0 & 0 & 0 & 973 & 1014 & 0 & 0 & & 5 \\
\hline GRANIZADA & 0 & 0 & 268 & 43 & 0 & 0 & 491 & 230 & 7 & 3 & 208 & 320 & 435 \\
\hline HELADA & 0 & 0 & 0 & 0 & 3000 & 0 & 0 & 0 & 0 & 0 & 1228 & 0 & 0 \\
\hline $\begin{array}{l}\text { INCENDIO } \\
\text { FORESTAL }\end{array}$ & 9 & 4990 & 111 & 83 & 53 & 89 & & 0 & 0 & 80 & 31 & 0 & 4990 \\
\hline INUNDACIÓN & 81616 & 19181 & 45685 & 67034 & 123846 & 20368 & 114353 & 290657 & 344292 & 57644 & 563364 & 0 & 123903 \\
\hline MAREJADA & 0 & 0 & 0 & 0 & 0 & 0 & 1928 & 1928 & & 0 & 0 & 0 & 86 \\
\hline SISMO & 97 & 0 & 0 & 100 & 2786 & 227 & 0 & 2482 & 4386 & 27 & 33 & 3 & 1849 \\
\hline $\begin{array}{l}\text { TORMENTA } \\
\text { ELÉCTRICA }\end{array}$ & 0 & 0 & 0 & 0 & 0 & 0 & 0 & 0 & 0 & 1 & 15 & 82 & 149 \\
\hline VENDAVAL & 4312 & 13973 & 8021 & 11151 & 17918 & 171789 & 19293 & 14273 & 12892 & 17493 & 20410 & 34732 & 585599 \\
\hline SEQUÍA & 0 & 1609 & 6480 & 6251 & 8726 & 2376 & 300 & 4100 & 0 & 2463 & 382783 & & 5897 \\
\hline TOTAL ANUAL & 93273 & 40584 & 58677 & 80423 & 167818 & 209245 & 148596 & 316812 & 24610 & 85287 & 20027 & 43152 & 731774 \\
\hline
\end{tabular}

De la sistematización de víctimas en la ocurrencia de eventos naturales sobresale la capacidad reactiva del Estado. Para visibilizar dicha postura se puede hacer énfasis, a manera de ejemplo, en departamentos como Antioquia, Bolívar, Chocó, Cundinamarca, Santander y Tolima, los cuales entre los años 2007 a 2012 frente al evento inundación presentan un gran número de repeticiones que va aumentando anualmente, lo que contradice la finalidad preventiva de las normas en materia de gestión del riesgo, porque se considera que a mayor número de repeticiones del evento en determinado territorio el Estado adquiere y especializa las potencialidades que le deberían proporcionar mayor y mejor capacidad de función a través de estudios de amenazas y que deriven en un diagnóstico que determine como podría ir evolucionando y sobreviniendo el fenómeno, de ahí que lo que se entre a evitar no sea la repetición del evento sino las consecuencias que éste puede generar a su paso en términos de las afectaciones a la población en situación de vulnerabilidad. Es de anotar que departamentos como Antioquia y Cundinamarca cuentan con una buena capacidad técnica y financiera para la ejecución de planes y proyectos sobre la gestión del riesgo; pese a dichas bondades, estos departamentos son los que más registros aportan al consolidado histórico nacional respecto de los eventos inundación, deslizamiento y avalancha.

\section{Conclusiones}

La prevención del riesgo en Colombia viene siendo impuesta como materia en el sistema de planeación regional, departamental y municipal desde la década de los años ochenta, lo que acredita la experticia administrativa que deben haber adquirido las entidades territoriales sobre este componente. La inclusión de la gestión del riesgo en la planeación territorial fue ampliada y especializada por la Ley 1523 de 2012, de donde se derivan las siguientes obligaciones para el orden administrativo territorial del Estado colombiano:

- Elaboración de planes de gestión del riesgo de desastres y estrategia de respuesta de emergencias en Departamentos y Municipios, los cuales se integran a los Planes de Ordenamiento Territorial, de manejo de cuencas y a los planes de desarrollo departamentales, distritales y municipales. 
- Obligación de incorporar el componente de reducción del riesgo en la formulación y elaboración de planes, programas y proyectos de desarrollo regional y urbano.

- Los planes de ordenamiento territorial, de manejo de cuencas hidrográficas y de planificación del desarrollo en los diferentes niveles de gobierno, deberán integrar el análisis del riesgo en el diagnóstico biofísico, económico y socioambiental y, considerar el riesgo de desastres, como un condicionante para el uso y la ocupación del territorio, procurando de esta forma evitar la configuración de nuevas condiciones de riesgo.

- Dentro del año siguiente a la entrada en vigencia de la Ley 1523 de 2012, las entidades territoriales municipales y distritales debieron revisar y ajustar los planes de ordenamiento territorial a la gestión del riesgo, lo mismo que los planes de desarrollo.

De los anteriores que como parte peticionaria veamos la necesidad que la Honorable CIDH inste al Estado de Colombia a presentar las disposiciones legales y/o reglamentarias o las directrices de política para la realización de inventarios de asentamientos en riesgo, de manera que las entidades territoriales cuenten con el soporte de legalidad previsto para el ejercicio de sus funciones; a presentar las disposiciones legales y/o reglamentarias o las directrices de política para el señalamiento, la delimitación y definición del tratamiento de las zonas expuestas a amenazas derivadas de fenómenos naturales, socio naturales, o antropogénicos no intencionales; definir cuáles son los mecanismos reglamentarios de reubicación de asentamientos, y con ello, las normas sobre procesos de reasentamiento poblacional a raíz de la gestión del riesgo; las disposiciones legales y/o reglamentarias o las directrices de política para la efectiva reserva de tierras destinadas exclusivamente a los reasentamientos poblacionales por eventos naturales enmarcados como emergencia o riesgo por el SNGRD, y las condiciones de uso de la adquisición y expropiación de inmuebles, sin que con ello se atente contra la autonomía municipal que reconoce nuestro orden constitucional.

\section{Bibliografía}

Asamblea General de la Organización de las Naciones Unidas (1991) Resolución 46/182, por medio de la cual se aprueba el fortalecimiento de la coordinación de la asistencia humanitaria de emergencia del sistema de las Naciones Unidas. Nueva York: Asamblea General de la Organización de las Naciones Unidas.

Asamblea General de la Organización de las Naciones Unidas (1989) Resolución 44/236, por medio de la cual se aprueba el Decenio Internacional para la Reducción de los Desastres Naturales. Nueva York: Asamblea General de la Organización de las Naciones Unidas.

Asamblea General de la Organización de las Naciones Unidas (1982) Carta Mundial de la Naturaleza, Resolución 37/7. Nueva York: Asamblea General de la Organización de las Naciones Unidas.

Asamblea general de la Organización de las Naciones Unidas (2005). Declaración de Mauricio y Estrategia de Mauricio para la ejecución ulterior del Programa de Acción para el desarrollo sostenible de los pequeños Estados insulares en desarrollo. Puerto Luis (Mauricio): Asamblea General de la Organización de las Naciones Unidas

Conferencia de las Naciones Unidas sobre el Medio Humano (1972) Conferencia de Estocolmo. Estocolmo: Organización de las Naciones Unidas.

Conferencia de las Naciones Unidas sobre el Medio Ambiente y el Desarrollo (1992) Declaración de Rio de Janeiro sobre el Medio Ambiente y el Desarrollo. Rio de Janeiro: Organización de las Naciones Unidas.

Conferencia de las Naciones Unidas sobre sistemas de alerta temprana (2003). Conferencia Internacional sobre sistemas de alerta temprana. Bonn (Alemania): Organización de las Naciones Unidas.

Conferencia Mundial sobre la Reducción de Desastres (2005) Informe de la Conferencia Mundial sobre la Reducción de los Desastres. A/CONF.206.6. Hyogo: Organización de las Naciones Unidas. 
Conferencia Mundial sobre la Reducción de los Desastres Naturales (1994) Informe de la Conferencia Mundial sobre la Reducción de los Desastres Naturales. A/CONF.172/9. Yokohama: Organización de las Naciones Unidas.

Congreso de la República de Colombia (1979). Ley No. 9. Por la cual se dictan Medidas Sanitarias. Diario Oficial No. 35308, del 16 de julio de 1979. Congreso de la República.

Congreso de la República de Colombia (1988). Ley No. 46. Por la cual se crea y organiza el sistema nacional para la prevención y atención de desastres, se otorga facultades extraordinarias al presidente de la república y se dictan otras disposiciones. Diario Oficial No 38.559, del 2 de noviembre de 1988. Congreso de la República.

Congreso de la República de Colombia (1989) Ley 9, por la cual se dictan normas sobre planes de desarrollo municipal, compraventa y expropiación de bienes y se dictan otras disposiciones.

Congreso de la República de Colombia. (1997). Ley 388 de 1997. Por la cual se modifica la Ley 9 de 1989, y la Ley 2 de 1991 y se dictan otras disposiciones. Diario Oficial 43.091 del 18 de julio de 1997.

Congreso de la República de Colombia. (2011). Ley 1454.

Congreso de la República de Colombia (2012). Ley 1523. Por la cual se adopta la política nacional de gestión del riesgo de desastres y se establece el Sistema Nacional de Gestión del Riesgo de Desastres y se dictan otras disposiciones. Diario Oficial 48.411 del 24 de abril de 2012.

Congreso de la República de Colombia. (2015). Ley 1753.

Constitución Nacional de Colombia (1991).

Corte Constitucional de la República de Colombia (1992). Sentencia T - 411. Magistrado ponente: Alejandro Martínez Caballero. Bogotá: Corte Constitucional.

Corte Constitucional de la República de Colombia (2010). Sentencia C - 595. Magistrado ponente: Jorge Iván Palacio Palacio. Bogotá: Corte Constitucional.

Corte Constitucional de la República de Colombia (2011). Sentencia T - 724. Magistrado ponente: Nilson pinilla Pinilla. Bogotá: Corte Constitucional.

Cumbre Mundial sobre Desarrollo Sustentable (2002) Declaración de Johannesburgo sobre Desarrollo Sustentable. Johannesburgo: Organización de las Naciones Unidas.

Ministerio de Justicia (1970). Decreto 1355. Por el cual se dictan las normas de policía. Diario Oficial No 33.139, del 4 de septiembre de 1970. Congreso de la República.

MORCILLO DOSMAN, P. P. (2007) Derecho urbanístico colombiano. Historia, derecho y gestión. Bogotá: Temis.

Presidencia de la República de Colombia (1974). Decreto 2811. Por el cual se dicta el Código Nacional de Recursos Naturales Renovables y de Protección al Medio Ambiente.

Presidencia de la República de Colombia (1986). Decreto Ley 1222. Por el cual se expide el Código de Régimen Departamental.

Presidencia de la República de Colombia (1986). Decreto Ley 1333. Por el cual se expide el Código de Régimen Municipal. 
Presidencia de la República de Colombia (1989). Decreto Ley No. 919. Por cual se organiza el Sistema Nacional para la Prevención y Atención de Desastres y se dictan otras disposiciones. Diario Oficial No 38.799, del 1 de mayo de 1989.

SÁNCHEZ STEINER, L. M. (2008) Éxodos rurales y urbanización en Colombia. Perspectiva histórica y aproximaciones teóricas. En: Revista Bitácora N. 13. Bogotá: Universidad Nacional de Colombia. pp. 57 -72 .

VICTORIA MORALES, M. I. \& MOLINA PRIETO, C. A. (2003) Reasentamiento involuntario: Integración y civilización. En: Revista Bitácora N. 7. Bogotá: Universidad Nacional de Colombia. pp. $19-25$.

VIVIESCAS, F. (1989) Urbanización y ciudad en Colombia. Bogotá: Foro Nacional por Colombia.

ZAMBRANO PANTOJA, F. R. (2013) Desarrollo urbano en Colombia: Una perspectiva histórica. Seminario Políticas e instituciones para el desarrollo urbano futuro en Colombia. Bogotá, Departamento Nacional de Planeación. 